\title{
IMPACT OF INTRACORONARY ADENOSINE ADMINISTRATION DURING PRIMARY PERCUTANEOUS CORONARY INTERVENTION
}

\author{
By
}

\author{
Abd El-Rahman El-Sayed Metwally, Mamdouh El-Tahan, Moustafa \\ Mokarrab, Tarek Bassiony and Mohamed El-Shorbagy*
}

Cardiovascular department, Faculty of Medicine, Al-Azhar University, Cairo, Egypt, ${ }^{*}$ clinical pathology department

Corresponding author: Abd El-Rahman El-Sayed Metwally

E-mail: dr_abdalrahman86@yahoo.com

\begin{abstract}
Background: Currently myocardial reperfusion with p-PCI is the best treatment strategy for STEMI, However, myocardial perfusion at the cellular level remains impaired despite removal of coronary obstruction in up to 50\% of STEMI patients Several methods have been evaluated to improve reperfusion, including heart rate reduction, aspiration thrombectomy and several pharmacological approaches, such as glycoprotein platelet inhibitors, adenosine and drugs able to dilate the microcirculation, seem to be most effective when locally delivered through IC injection, probably because this results in an increased drug bioavailability in the area at risk.
\end{abstract}

Objective: the aim of our study was to clarify the efficacy of IC adenosine versus standard therapy only in STEMI patients undergoing $\mathrm{p}$-PCI.

Patients and methods: Selected 45 patients presented to emergency room by acute STEMI and were randomized into two groups: Control group (25 patients) and Patient group who received IC adenosine (20 patients). Both were evaluated during p-PCI by MBG and TIMI flow grade, after the procedure, both groups was evaluated by STR in ECG, cardiac enzymes at 0-6-12 hours, and echocardiography within 24 hours and after 40 days.

Results: in patient group, TIMI flow was significantly better, incidence of ST resolution was significantly higher, and level of cardiac enzymes was significantly higher at 6 hours and significantly lower at 12 hours. Moreover, we found a larger increase in LVEF - and subsequently reduction in the incidence of heart failure, more improvement of MR, TR, PASP and TAPSE in patient group.

Conclusion: This study clarified clinical benefits for IC adenosine in hard endpoints, such as TIMI flow, percentage of LVEF improvement, in patients undergoing p-PCI.

Key words: Adenosine, p-PCI, myocardial perfusion.

\section{ABBREVIATIONS}

BMI: body mass index - FMC: first medical contact - IC: intracoronary - IRA: infarct related artery - MBG: myocardial blush grade - MR: mitral regurgitation - MW: MannWhitney Test - PASP: Pulmonary artery systolic pressure- p-PCI: primary percutaneous coronary intervention - STEMI: ST- segment elevation myocardial infarction STR: ST segment resolution - TAPSE: Tricuspid annular plane systolic excursion TIMI: thrombolysis in myocardial infarction TR: tricuspid regurgitation. 


\section{INTRODUCTION}

Currently myocardial reperfusion with p-PCI is the best treatment strategy for STEMI (O'Gara et al., 2013). However, myocardial perfusion at the cellular level remains impaired despite removal of coronary obstruction in up to $50 \%$ of STEMI patients, the current factors, embolization of coronary thrombus into the distal vasculature, micro vascular plugging, vasospasm, interstitial edema, local inflammation, and cellular injury play a role (Niccoli et al., 2009). Several methods have been evaluated to improve reperfusion, including heart rate reduction, aspiration thrombectomy and several pharmacological approaches, such as glycoprotein platelet inhibitors, adenosine and drugs able to dilate the microcirculation (De Rosa et al., 2014). These pharmacologic approaches seem to be most effective when locally delivered through IC injection, probably because this results in an increased drug bioavailability in the area at risk (Zhao, et al., 2014). Endogenous adenosine plays an important role in maintaining myocardial perfusion through its potent vasodilator effect and NO-inducing proprieties, as well as its anti-inflammatory and antiplatelet properties. Interestingly, Bune et al. (2015) have recently shown in an animal model that administration of ADPthat is largely converted to adenosine by endothelial cells in the blood stream- is indeed able to substantially limit the final infarct size and that this effect is at least in part related to an increased release of $t$ PA. The use of adenosine in patients with STEMI has been tested in previous studies, but results are conflicting because of small sample sizes, different dosages or administration routes (Singh, et al. 2012).
The aim of the present study was to clarify the effect of IC adenosine versus placebo on clinical outcomes in patients with STEMI undergoing p-PCI.

\section{PATIENTS AND METHODS}

Our study includes 45 patients presented by acute STEMI divided into two groups Patients group include 20 patients who received IC Adenosine, while Control group include 25 patients who received the standard therapy. The following patients were excluded: if thrombolytic therapy was given, with previous history of CAD, Previous heart failure, Congenital heart diseases, cardiac surgery, permanent AF, if receiving oral anticoagulation, Allergy to Adenosine, or if patients with poor Echocardiographic window.

All patients were subjected to Informed consent, Complete history as regard risk factors, Heart failure symptoms, Drug history, Full physical examination ,Blood sample for CBC especially hemoglobin level, serum creatinine, RBS, INR, LDL, HDL, Triglyceride, resting 12 leads ECG within 10 minutes from FMC and after 90 minutes from p-PCI to assess the STR \%, p-PCI within 90 minutes from FMC with \& Without adenosine Pretreatment, Patients who were randomized to the adenosine group received (100 mic in case of left system CAD, and 50 mic in case of RCA occlusion) of adenosine (diluted into $5 \mathrm{~mL}$ of normal saline) through the guiding catheter into the culprit coronary artery after aspiration of the present thrombi (if applicable) and prior to stenting. The rest of the intervention strategy, including use of glycoprotein $\mathrm{IIb} /$ IIIa inhibitors and drug-eluting stents, were done once indicated. Patients who 
were randomized to the standard therapy did not received adenosine pretreatment, and the procedure was carried out in the usual manner. Administration of adenosine for treatment of no-reflow phenomenon during the procedure was allowed in both groups. Estimation of the perfusion degree were done by TIMI flow grade and myocardial Blush grade (MBG).All patients received a Clopidogrel Tablet $600 \mathrm{mg}$ PO, ASA 300mg PO \& Enoxaparin $1 \mathrm{mg} / \mathrm{kg}$ at FMC. Post-procedural antiplatelet regimen consisted of aspirin $81 \mathrm{mg}$ p.o /day indefinitely and Clopidogrel $150 \mathrm{mg}$ p.o/day for 7-14 day then $75 \mathrm{mg}$ PO daily for one year. Full study Transthoracic Echocardiography (TTE) was performed immediate after p-PCI within 24 hours and another study was done after 40 days apart from the procedure, especially for assessment of the LV EF \%, Grade of MR,TR,-if present-TAPSE and PASP.

\section{Statistical analysis:}

Data were analyzed using Statistical Program for Social Science (SPSS) version 24. Quantitative data were expressed as mean \pm standard deviation (SD). Qualitative data were expressed as frequency and percentage. Mean (average): is the central value of a discrete set of numbers, specifically the sum of values divided by the number of values. Standard deviation (SD): is the measure of dispersion of a set of values, a low SD indicates that the values tend to be close to the mean of the set, while a high SD indicate that the values are spread out over a wider range. Median is the value separating the higher half from the lower half of data. The basic advantage of median comparing to mean is that the median is not skewed so much by small proportion of extremely large or small values. IQR is the measure of statistical dispersion, being equal to the difference between 75 th and 25 th percentile. The following tests were done: Independentsamples t-test of significance: was used when comparing between two means. Mann -Whitney U test: was used when comparing between two means (for abnormal distributed data). Chi-square test: was used when comparing between non-parametric data. Probability (Pvalue): $\mathrm{P}$-value $<0.05$ was considered significant.

\section{RESULTS}

- The following tables represent comparison between patient and control groups:

- There was no statistical significant difference ( $p$-value > 0.05) between studied groups as regard risk factors (age, sex, DM, HTN, dyslipidemia, smoking, family history and BMI) (Table 1). 
Table (1): Comparison between studied groups as regard demographic criteria and risk factors

\begin{tabular}{|c|c|c|c|c|c|c|}
\hline Risk factors & Groups & \multirow{2}{*}{\multicolumn{2}{|c|}{$\begin{array}{c}\begin{array}{c}\text { Patients } \\
(\mathrm{N}=20)\end{array} \\
56.9 \pm 8.5\end{array}$}} & \multirow{2}{*}{\multicolumn{2}{|c|}{$\begin{array}{c}\begin{array}{c}\text { Control } \\
(\mathrm{N}=\mathbf{2 5})\end{array} \\
\mathbf{5 5 . 6} \pm \mathbf{1 1 . 5}\end{array}$}} & P-value \\
\hline Age & Mean \pm SD & & & & & 0.678 \\
\hline \multirow{2}{*}{ Sex } & M & 16 & $80 \%$ & 23 & $92 \%$ & \multirow{2}{*}{0.239} \\
\hline & $\mathbf{F}$ & 4 & $20 \%$ & 2 & $8 \%$ & \\
\hline \multirow{2}{*}{ DM } & No & 11 & $55 \%$ & 19 & $76 \%$ & \multirow{2}{*}{0.138} \\
\hline & Yes & 9 & $45 \%$ & 6 & $24 \%$ & \\
\hline \multirow{2}{*}{ HTN } & No & 11 & $55 \%$ & 14 & $56 \%$ & \multirow{2}{*}{0.947} \\
\hline & Yes & 9 & $45 \%$ & 11 & $44 \%$ & \\
\hline \multirow{2}{*}{ Dyslipidemia } & No & 12 & $60 \%$ & 8 & $32 \%$ & \multirow{2}{*}{0.06} \\
\hline & Yes & 8 & $40 \%$ & 17 & $68 \%$ & \\
\hline \multirow{2}{*}{ Smoking } & No & 9 & $45 \%$ & 8 & $32 \%$ & \multirow{2}{*}{0.371} \\
\hline & Yes & 11 & $55 \%$ & 17 & $68 \%$ & \\
\hline \multirow{2}{*}{$\begin{array}{l}\text { Family } \\
\text { history }\end{array}$} & No & 18 & $90 \%$ & 25 & $100 \%$ & \multirow{2}{*}{0.106} \\
\hline & Yes & 2 & $10 \%$ & $\mathbf{0}$ & $0 \%$ & \\
\hline BMI & Mean \pm SD & \multicolumn{2}{|c|}{$29.1 \pm 4.6$} & \multicolumn{2}{|c|}{$28.9 \pm 4.1$} & $>0.05$ \\
\hline
\end{tabular}

- Catheterization data of both groups as regard type of STEMI presentation, IRA, single versus multi vessel disease and complete versus staged PCI (Table 2).

Table (2): Description of catheterization data in both groups

\begin{tabular}{|c|c|c|c|c|c|}
\hline \multicolumn{2}{|c|}{ Catheterization data } & \multicolumn{2}{|c|}{$\begin{array}{c}\text { Control group } \\
(\mathbf{N}=\mathbf{2 5})\end{array}$} & \multicolumn{2}{|c|}{$\begin{array}{c}\text { Patients group } \\
(\mathbf{N}=\mathbf{2 0})\end{array}$} \\
\hline \multirow{9}{*}{$\begin{array}{c}\text { Presentation } \\
\text { type } \\
\text { Of STEMI }\end{array}$} & Anterior & 12 & $48 \%$ & 10 & $50 \%$ \\
\hline & Antero- Lateral & 1 & $4 \%$ & $\mathbf{0}$ & $0 \%$ \\
\hline & Antero- inferior & $\mathbf{0}$ & $0 \%$ & 1 & $5 \%$ \\
\hline & Inferior & 7 & $28 \%$ & 5 & $25 \%$ \\
\hline & Infero -Posterior & 3 & $12 \%$ & 1 & $5 \%$ \\
\hline & Infero -Lateral & 1 & $4 \%$ & $\mathbf{0}$ & $0 \%$ \\
\hline & Postero-Lateral & 1 & $4 \%$ & $\mathbf{0}$ & $\mathbf{0 \%}$ \\
\hline & Lateral & $\mathbf{0}$ & $0 \%$ & 2 & $10 \%$ \\
\hline & Posterior & $\mathbf{0}$ & $0 \%$ & 1 & $5 \%$ \\
\hline \multirow{4}{*}{ IRA } & LAD & 12 & $48 \%$ & 11 & $55 \%$ \\
\hline & LCX & 3 & $12 \%$ & 2 & $10 \%$ \\
\hline & $\mathbf{O M}$ & 2 & $8 \%$ & 1 & $5 \%$ \\
\hline & RCA & 8 & $32 \%$ & 6 & $30 \%$ \\
\hline \multicolumn{2}{|c|}{ Single vessel disease } & 12 & $48 \%$ & 8 & $40 \%$ \\
\hline \multicolumn{2}{|c|}{ Multi vessel disease } & 13 & $52 \%$ & 12 & $60 \%$ \\
\hline \multirow{2}{*}{$\begin{array}{c}\text { Complete } \\
\text { revascularization }\end{array}$} & No & 15 & $60 \%$ & 12 & $60 \%$ \\
\hline & Yes & 10 & $40 \%$ & 8 & $40 \%$ \\
\hline \multirow{2}{*}{ Staged PCI } & No & 21 & $84 \%$ & 14 & $70 \%$ \\
\hline & Yes & 4 & $16 \%$ & 6 & $30 \%$ \\
\hline
\end{tabular}

- There was statistically significant difference ( $p$-value $<0.05$ ) between studied groups as regard TIMI flow but no statistical significant difference $(\mathrm{p}$ - value > 0.05) between both studied groups as regard $\mathrm{MBG}$ also there was no cases with no-reflow (Table 3). 
Table (3): Comparison between studied groups as regard TIMI flow, MBG and no reflow

\begin{tabular}{|c|c|c|c|c|c|c|}
\hline $\begin{array}{l}\text { Reperfusion } \\
\text { parameter }\end{array}$ & Groups & & $\begin{array}{l}\text { ents } \\
20)\end{array}$ & & & P-value \\
\hline \multirow{2}{*}{ TIMI flow } & II & 0 & $0 \%$ & 9 & $36 \%$ & \multirow{2}{*}{0.003} \\
\hline & III & 20 & $100 \%$ & 16 & $64 \%$ & \\
\hline \multirow{2}{*}{ MBG } & II & 2 & $10 \%$ & 6 & $24 \%$ & \multirow{2}{*}{0.222} \\
\hline & III & 18 & $90 \%$ & 19 & $76 \%$ & \\
\hline \multirow{2}{*}{ No reflow } & No & 20 & $100 \%$ & 25 & $100 \%$ & \multirow[t]{2}{*}{ - } \\
\hline & Yes & 0 & $0 \%$ & $\mathbf{0}$ & 0\% & \\
\hline
\end{tabular}

- There was statistically significant difference between studied groups as regard \% of STR(ST segment resolution) in ECG, Hs Troponin $\mathrm{T}$ level (after 6 hours and after 12 hours)
(P value <0.05) but no statistical significant difference between studied groups as regard Hs Troponin $\mathrm{T}$ level at 0 hours, or CKMB at $0,6,12$ hours ( $p$-value > 0.05) (Table 4).

Table (4): Comparison between studied groups as regard STR\%, Hs Troponin T, and CKMB levels

\begin{tabular}{|c|c|c|c|c|c|}
\hline \multicolumn{3}{|c|}{$\begin{array}{ll}\text { Cardiac enzymes } & \text { Groups } \\
\end{array}$} & $\begin{array}{l}\text { Patients } \\
(\mathbf{N}=\mathbf{2 0})\end{array}$ & $\begin{array}{l}\text { Control } \\
(\mathbf{N}=25)\end{array}$ & $\begin{array}{c}\text { P- } \\
\text { value }\end{array}$ \\
\hline \multirow{2}{*}{\multicolumn{2}{|c|}{ STR \% }} & Median & $75 \%$ & $62 \%$ & \multirow{2}{*}{0.001} \\
\hline & & IQR & $18.5(68.5-87)$ & $17(50-67)$ & \\
\hline \multirow{6}{*}{ 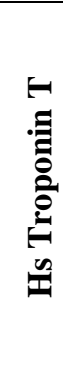 } & \multirow[b]{2}{*}{0 hour } & Median & 47 & 47 & \multirow[b]{2}{*}{$\begin{array}{c}0.918 \\
\text { NS }\end{array}$} \\
\hline & & IQR & $\begin{array}{c}896.5 \\
(29-925.5)\end{array}$ & $\begin{array}{c}1363 \\
(26-1389.5)\end{array}$ & \\
\hline & \multirow[b]{2}{*}{$\begin{array}{c}6 \\
\text { hours }\end{array}$} & Median & 6492 & 4829 & \multirow[b]{2}{*}{0.028} \\
\hline & & IQR & $\begin{array}{c}4864.5 \\
(4906-9770.5)\end{array}$ & $\begin{array}{c}4937 \\
(2436.5-7373.5)\end{array}$ & \\
\hline & \multirow[b]{2}{*}{$\begin{array}{c}12 \\
\text { hours }\end{array}$} & Median & 1594 & 3111 & \multirow[b]{2}{*}{0.01} \\
\hline & & IQR & $\begin{array}{c}1970 \\
(574.5-2445)\end{array}$ & $\begin{array}{c}6535 \\
(1600.5-8135.5)\end{array}$ & \\
\hline \multirow{6}{*}{$\sum_{v}^{v}$} & \multirow[b]{2}{*}{0 hour } & Median & 5 & 5 & \multirow[b]{2}{*}{0.801} \\
\hline & & IQR & $\begin{array}{c}44.25 \\
(3.25-47.5)\end{array}$ & $\begin{array}{c}43.5 \\
(3-46.5)\end{array}$ & \\
\hline & \multirow{2}{*}{$\begin{array}{c}6 \\
\text { hours }\end{array}$} & Median & 300 & 132 & \multirow[b]{2}{*}{0.058} \\
\hline & & IQR & $\begin{array}{c}197.5 \\
(102.5-300) \\
\end{array}$ & $\begin{array}{c}264 \\
(36-300) \\
\end{array}$ & \\
\hline & \multirow{2}{*}{$\begin{array}{c}12 \\
\text { hours }\end{array}$} & Median & 50 & 93 & \multirow[b]{2}{*}{0.102} \\
\hline & & IQR & $\begin{array}{c}56.75 \\
(18.25-75)\end{array}$ & $\begin{array}{c}170 \\
(29.5-199.5)\end{array}$ & \\
\hline
\end{tabular}

- There was statistically significant difference ( $p$-value $<0.05$ ) between studied groups as regard MR, TAPSE $\&$ TR ,but no statistical significant difference ( $p$-value > 0.05) between studied groups as regard EF and PASP (within 24 hour) (Table 5). 
Table (5): Comparison between studied groups as regard TTE (within 24 hours)

\begin{tabular}{|c|c|c|c|c|c|c|c|}
\hline \multicolumn{2}{|c|}{$\begin{array}{ll}\text { TTE } & \text { Groups } \\
\end{array}$} & \multicolumn{2}{|c|}{$\begin{array}{l}\text { Patients } \\
(\mathbf{N}=20)\end{array}$} & \multicolumn{2}{|c|}{$\begin{array}{l}\text { Control } \\
(\mathrm{N}=25)\end{array}$} & Test & $P$-value \\
\hline \multirow{2}{*}{$\mathbf{E F}$} & Mean & \multicolumn{2}{|c|}{44.8} & \multicolumn{2}{|c|}{41.3} & \multirow{2}{*}{0.95} & \multirow{2}{*}{0.346} \\
\hline & \pm SD & \multicolumn{2}{|c|}{12.8} & \multicolumn{2}{|c|}{11.8} & & \\
\hline \multirow{4}{*}{ MR } & No MR & 8 & $40 \%$ & 11 & $44 \%$ & \multirow{4}{*}{$\begin{array}{l}X^{2}= \\
12.1\end{array}$} & \multirow{4}{*}{0.007} \\
\hline & grade I & 7 & $35 \%$ & 7 & $28 \%$ & & \\
\hline & grade II & 5 & $25 \%$ & $\mathbf{0}$ & $0 \%$ & & \\
\hline & grade III & $\mathbf{0}$ & $0 \%$ & 7 & $28 \%$ & & \\
\hline \multirow{3}{*}{ TR } & No TR & 14 & $70 \%$ & 12 & $48 \%$ & \multirow{3}{*}{$\begin{array}{c}X^{2}= \\
7.6\end{array}$} & \multirow{3}{*}{0.023} \\
\hline & grade I & 5 & $25 \%$ & 3 & $12 \%$ & & \\
\hline & grade II & 1 & $5 \%$ & 10 & $40 \%$ & & \\
\hline \multirow{2}{*}{ TAPSE } & Normal & 18 & $90 \%$ & 11 & $44 \%$ & \multirow{2}{*}{$\begin{array}{l}X^{2}= \\
10.3\end{array}$} & \multirow{2}{*}{0.001} \\
\hline & Impaired & 2 & $10 \%$ & 14 & $56 \%$ & & \\
\hline \multirow[b]{2}{*}{ PASP } & Median & \multicolumn{2}{|c|}{24} & \multicolumn{2}{|c|}{27} & \multirow{2}{*}{$\begin{array}{c}M W= \\
195\end{array}$} & \multirow[b]{2}{*}{0.207} \\
\hline & IQR & \multicolumn{2}{|c|}{$\begin{array}{c}7.75 \\
(22.25-30)\end{array}$} & \multicolumn{2}{|c|}{$\begin{array}{c}6 \\
(25-31)\end{array}$} & & \\
\hline
\end{tabular}

- There was statistically significant difference ( $\mathrm{p}$-value $<0.05$ ) between studied groups as regard LVEF\%, MR,
TR, TAPSE \& PASP (after 40 days follow up) (Table 6).

Table (6): Comparison between studied groups as regard TTE (after 40 days)

\begin{tabular}{|c|c|c|c|c|c|c|}
\hline \multicolumn{2}{|c|}{$\begin{array}{ll}\text { TTE } & \text { Groups } \\
\end{array}$} & \multicolumn{2}{|c|}{$\begin{array}{l}\text { Patients } \\
(\mathbf{N}=\mathbf{2 0})\end{array}$} & \multicolumn{2}{|c|}{$\begin{array}{l}\text { Control } \\
(\mathrm{N}=25)\end{array}$} & P-value \\
\hline \multirow{2}{*}{ EF } & Mean & \multicolumn{2}{|c|}{ 53.5 } & \multicolumn{2}{|c|}{43.5} & \multirow{2}{*}{0.006} \\
\hline & \pm SD & \multicolumn{2}{|c|}{10.2} & \multicolumn{2}{|c|}{12.4} & \\
\hline \multirow{4}{*}{ MR } & No MR & 16 & $80 \%$ & 11 & $44 \%$ & \multirow{4}{*}{0.041} \\
\hline & Grade I & 2 & $10 \%$ & 7 & $28 \%$ & \\
\hline & Grade II & 2 & $10 \%$ & 2 & $8 \%$ & \\
\hline & Grade III & $\mathbf{0}$ & $0 \%$ & 5 & $20 \%$ & \\
\hline \multirow{3}{*}{ TR } & No TR & 17 & $85 \%$ & 14 & $56 \%$ & \multirow{3}{*}{0.011} \\
\hline & Grade I & 3 & $15 \%$ & 2 & $8 \%$ & \\
\hline & Grade II & $\mathbf{0}$ & $0 \%$ & 9 & $36 \%$ & \\
\hline \multirow{2}{*}{ TAPSE } & Normal & 19 & $95 \%$ & 13 & $52 \%$ & \multirow{2}{*}{0.002} \\
\hline & Impaired & 1 & $5 \%$ & 12 & $48 \%$ & \\
\hline \multirow[b]{2}{*}{ PASP } & Median & \multicolumn{2}{|c|}{23.5} & \multicolumn{2}{|c|}{26} & \multirow[b]{2}{*}{0.015} \\
\hline & IOR & \multicolumn{2}{|c|}{$\begin{array}{c}4.25 \\
(22.25-26.5)\end{array}$} & \multicolumn{2}{|c|}{$\begin{array}{c}7.5 \\
(25-32.5)\end{array}$} & \\
\hline
\end{tabular}

- There was no statistical significant difference ( $\mathrm{p}$-value > 0.05) between studied groups as regard MACE (Table 7).

Table (7): Comparison between studied groups as regard peri-procedural MACE

\begin{tabular}{|c|c|c|c|c|c|c|c|}
\hline $\begin{array}{ll}\text { MACE } & \text { Groups } \\
\end{array}$ & \multicolumn{3}{|c|}{$\begin{array}{l}\text { Control } \\
(\mathrm{N}=25)\end{array}$} & \multicolumn{2}{|c|}{$\begin{array}{l}\text { Patient } \\
(\mathrm{N}=\mathbf{2 0})\end{array}$} & & P-value \\
\hline NO MACE & 21 & \multicolumn{2}{|c|}{$84 \%$} & 18 & \multicolumn{2}{|c|}{$90 \%$} & \multirow{5}{*}{$0.05 \%$} \\
\hline Survived from VF & $\mathbf{1}$ & $4 \%$ & \multirow{4}{*}{$16 \%$} & 1 & $5 \%$ & \multirow{4}{*}{$10 \%$} & \\
\hline pulmonary edema & 2 & $8 \%$ & & $\mathbf{0}$ & $0 \%$ & & \\
\hline $\mathbf{A F}$ & 1 & $4 \%$ & & $\mathbf{0}$ & $0 \%$ & & \\
\hline $\begin{array}{c}\text { Advanved transient } \\
\text { AV block }\end{array}$ & $\mathbf{0}$ & $0 \%$ & & 1 & $5 \%$ & & \\
\hline
\end{tabular}




\section{DISCUSSION}

The main findings of our study are that IC Adenosine: a) is effective in improving myocardial reperfusion and TIMI flow in STEMI patients undergoing p-PCI; b) favors a better left and right ventricular remodeling, as suggested by the significantly higher LVEF and TAPSE value and subsequently the lower incidence of heart failure, also better improvement in MR, TR \& PASP in the adenosine group; c) no statistically significant difference in the incidence of no reflow nor per procedural MACE. The clinical benefit observed with IC adenosine largely outweighs the increased incidence of transient atrio-ventricular block. Despite the "no reflow" is associated with a poor prognosis results from previous studies are conflicting and inconclusive. Several studies have investigated the benefits of the preventive use of adenosine during and after reperfusion therapy for acute myocardial infarction. These studies used different, sometimes complicated and timeconsuming, protocols of adenosine infusion during and after the PCI procedure or thrombolytic therapy.

Akturk et al. (2014) showed that IC Verapamil provides better TIMI flow in comparison with IC adenosine, so in this study they did not ignore the clinical benefits of IC adenosine despite better results of IC Verapamil which was beyond the scope of our study.

Mukesh et al. (2012) analyzed 7 studies involving 1030 participants who were treated with IC adenosine. They assessed mortality, heart failure, MACE, STR, LVEF \%, TIMI flow, MBG, and side effects, but were unable to draw definitive conclusions on any of the clinical outcomes. Their meta-analysis included one study used a nonplacebo (nitroglycerine) control group.

Polimeni et al. (2014) reported a metaanalysis of conference abstracts, which included 10 RCTs in which patients were treated with IC adenosine. They found that adenosine treatment improved major cardiovascular adverse events and heart failure rates in patients with STEMI treated with PCI. Their findings are partly consistent with our conclusions.

Stoel et al. (2008) investigated the influence of very-high-dose intracoronary adenosine (60 mg within 5 to 10 minutes) on persistent ST-segment elevation after primary PCI. Intracoronary adenosine accelerated ST segment resolution and recovery of micro vascular perfusion, as assessed by the TIMI frame count and MBG.

These results should not be misunderstood, and the golden STEMI rule "earlier is better" holds true for IC adenosine as for all other STEMI treatments. As for any study, some limitations should be acknowledged that are related to :1) different definitions in the studies for different endpoints ;2) some differences in the baseline characteristics found between the studies; $3)$ dose and method of adenosine administration were heterogeneous among the previous studies; 4) difficult patient compliance in the follow up period 5) given that our study and previous studies were designed on a relatively limited number of included patients, for this reason all results on hard clinical endpoints should be interpreted with caution. Further studies are needed to 
establish a cut-off to identify the optimal dose to be administered. Finally, the interaction between adenosine and concomitant treatments, such as antiplatelet agents, also deserves further attention.

\section{CONCLUSION}

Our study provides evidence that, besides increasing the reperfusion indices, IC adenosine is associated to a more favorable left ventricular remodeling, with larger increase in LVEF and lower incidence of heart failure and also can be given safely as an IC route.

\section{CONFLICT OF INTEREST STATEMENT}

The authors have no conflicts of interest to declare.

\section{ACKNOWLEDGMENTS}

Thanks to Dr Abdelmaksoud Ahmed, Dr. Hani Khalaf and Dr. Elsayed Elmerghany, Faculty of Medicine, Al Azhar University, Cairo,Egypt- Dr. Ahmed Bakry, Faculty of Medicine, South Valley University, Qena, Egypt- Dr. Ahmed Daod, Dr-Erfan \& Bagedo general Hospital, Jeddah, KSA, for their great efforts during doing this study.

\section{REFERENCES}

1. Akturk IF, Yalcin AA, Biyik I, Sarikamis C, Turhan N, Caglar and Erturk M. (2014): Effects of verapamil and Adenosine in an adjunct to tirofiban on resolution and prognosis of noreflow phenomenon in patients with acute myocardial infarction, Minerva Cardioangiol, 62: 389-397.
2. Bune LT., Larsen JR., Thaning P.,Bune NE., Rasmussen P. and Rosenmeier JB. (2013): adenosine diphosphate reduces infarct size and improves porcine heart function after myocardial infarct, Physiol. Rep. 1, e00003.

3. De Rosa S., Caiazzo G., Torella D., Indolfi C. (2014): Aspiration thrombectomy: an easily forgiven "latecomer", J. Am. Coll. Cardiol, 63: 2052-2053.

4. Mukesh S, Tejaskumar S and Kavia K. (2012): Safety and efficacy of intracoronary adenosine administration in patients with acute myocardial infarction undergoing primary percutaneous coronary intervention: a meta-analysis of randomized controlled trials. Ther Adv Cardiovasc Dis., 6:101-114.

5. Niccoli G., Burzotta F.,Galiuto L. and Crea F. (2009): Myocardial no-reflow in humans, J. Am. Coll. Cardiol, 54: 281-292.

6. O'Gara P, Kushner F, Ascheim $D$, Casey $D$, Chung $M$ and de Lemos J. (2013): ACCF/AHA guideline for the management of ST-elevation myocardial infarction: a report of the American College of Cardiology Foundation/American

Heart Association task force on practice guidelines, J. Am. Coll. Cardiol , 61:e78-e140. 
7. Polimeni A, De Rosa $S$ and Sorrentino S. (2014):

Intracoronary adenosine administration reduces MACE and heart failure in patients with acute myocardial infarction undergoing primary percutaneous coronary intervention: a meta-analysis of RCTs. Giornale Italiano di Cardiologia, 15(Suppl 1):e27.

8. Singh M., Shah T., Khosla K. and Singh P. (2012): Molnar J.,Khosla S.,Safety and efficacy of intracoronary adenosine administration in patients with acutemyocardial infarction undergoing primary percutaneous coronary intervention: a metaanalysis of randomized controlled trials, Ther. Adv. Cardiovasc. Dis, 6:101-114.

9. Stoel MG, Marques KM, de Cock CC, Bronzwaer JG, von Birgelen $C$ and Zijlstra $F$. (2008): High-dose Adenosine for suboptimal myocardial reperfusion after primary PCI: a randomized placebo controlled pilot study. Catheter Cardiovasc Interv , 71:283-289.

10. Zhao S., Qi G., Tian W., Chen L. and Sun Y. (2014): Effect of intracoronary nitroprusside in preventing no reflow phenomenon during primary percutaneous coronary intervention: a metaanalysis, J. Interv. Cardiol, 27: 356-364. 


\section{تأثير حقن الثريان التاجى بعقار الادينوسين اثناء اجراء دعامة

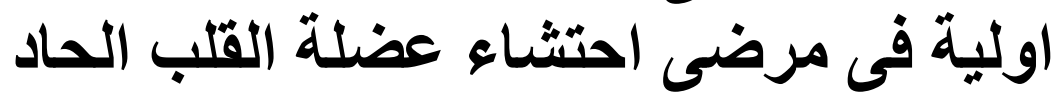

عبدالرحمن السيد متولي، ممدوح حلمي الطحان، مصطفى إبراهيم مقرب، طارق

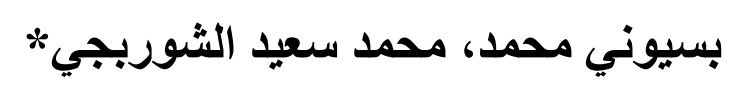

قسم القلب والأوعية الاموية، ثقسم الباثولوجيا الاكلينيكية، كلية الطب، جامعة الأزهر، القاهرة

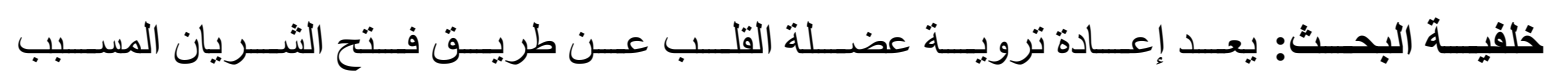

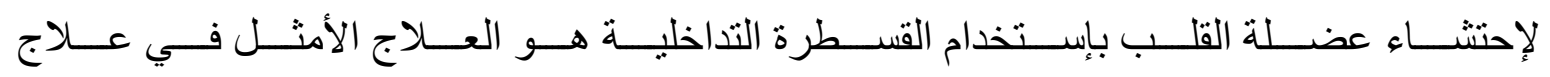

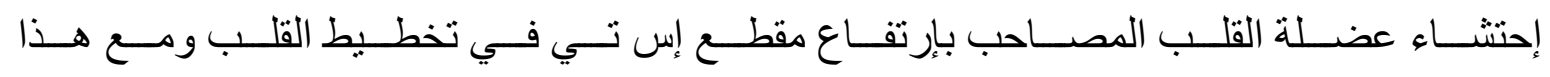

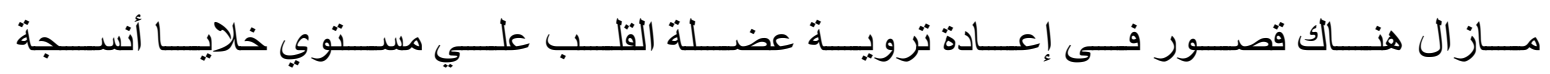

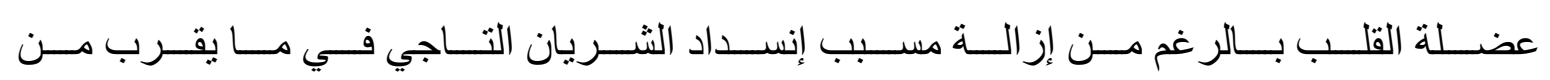
\% \% 50 من عد الحالات.

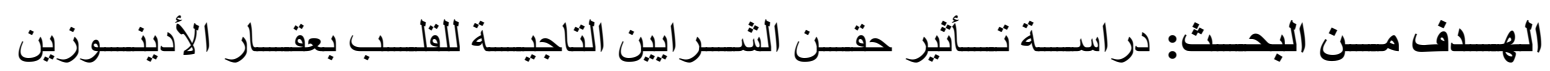

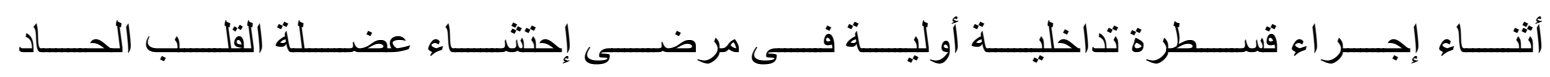
المصاحب لإرتفاع مقطع إس تي.

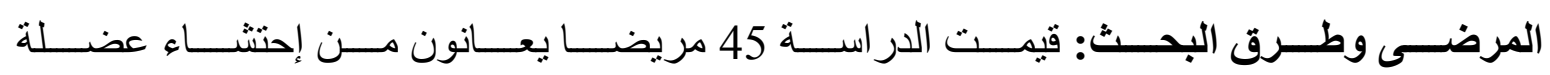

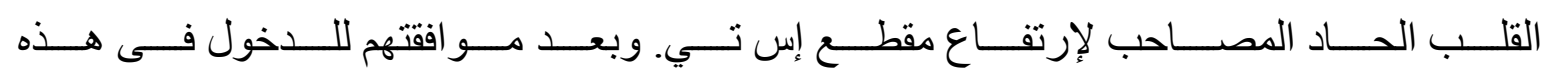

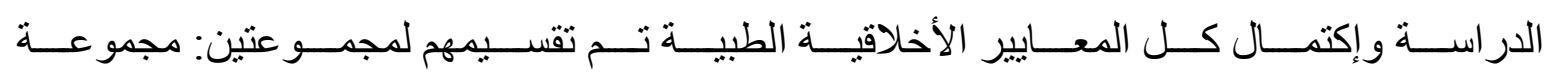

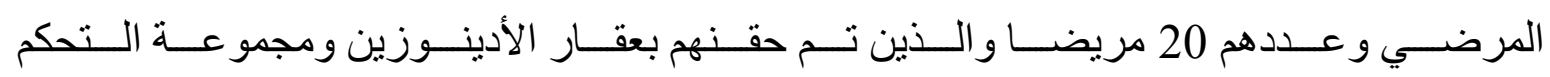
و عددهم 25 مريضا وكانت تتر اوح بين 18 و 75 عام.

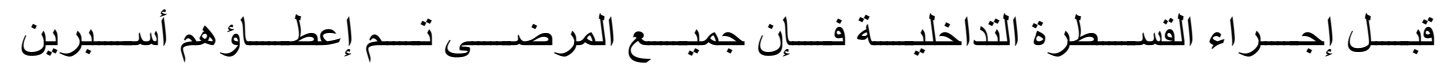

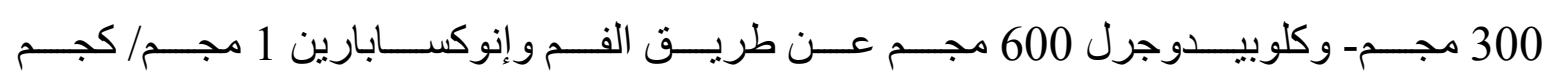

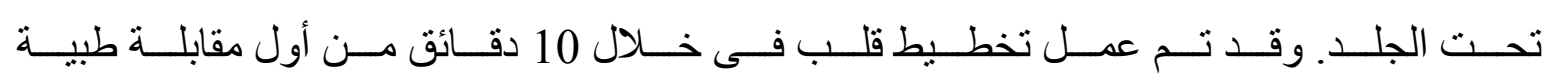
للمريض وتخطيط قلب أخر بعد الإنتهاء من القسطرة التداخلية ب 90 دقيقة.

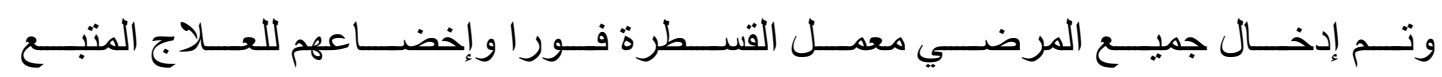

طبقا للتوصيات و الإرشادات العالمية. 


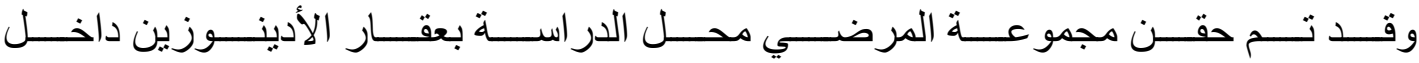

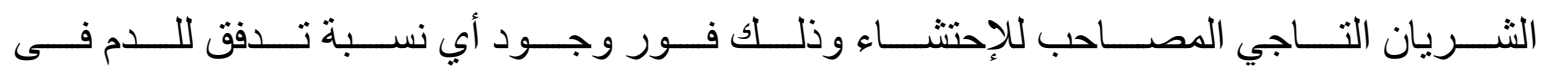

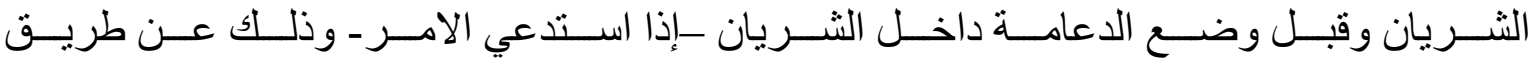

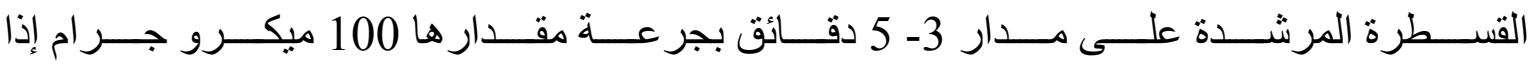

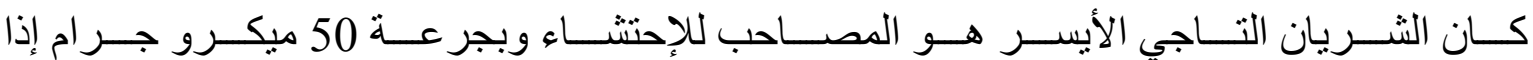
كان الثريان التاجي الأيمن هو المصاحب للإحتشاء.

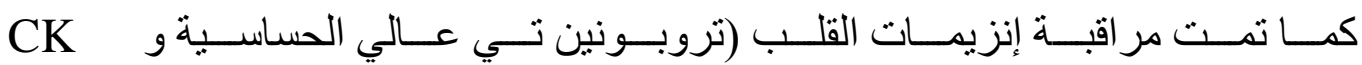
على مدار 0-6-6 ساعة من أول مقابلة طبية.

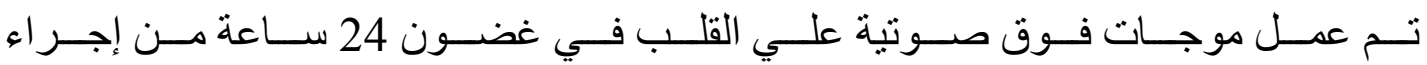
القسطرة التداخلية و أيضا بعد 40 يوم من حدوث إحتشاء عضلة القلب.

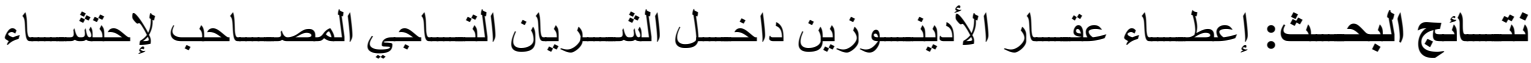

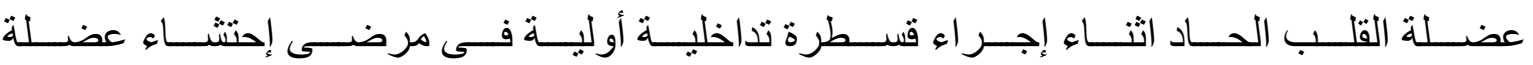

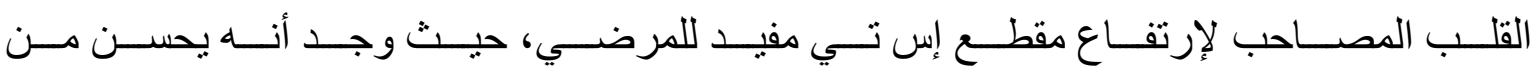

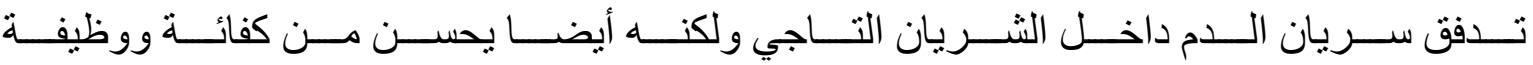

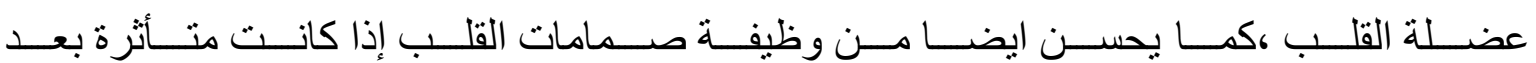
إحتشاء عضلة القلب.

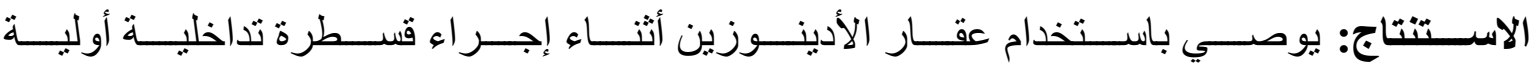

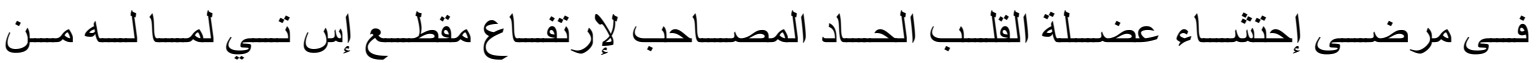
فو ائد عديدة على صحة المرضي. 